\title{
BLICKDIAGNOSE
}

Seit Jahren Wirbelgleiten

\section{Sprungschanze im Rücken}

\section{Ein 47 Jahre alter Patient stellt sich be- schwerdefrei wegen eines seit Jahren bekannten Wirbelgleitens im Bereich der Lendenwirbelsäule zur klinisch-radiolo- gischen Verlaufskontrolle vor. Er bittet um Beratung, welche Therapiemöglichkeiten bzw. -notwendigkeiten bestehen. Bei der körperlichen Untersuchung imponiert tief- lumbal eine deutliche Stufenbildung, ein sogenanntes Sprungschanzenphänomen.}

_ Die Spondylolyse ist eine Spaltbildung in der Interartikularportion des Wirbelbogens. In deren Folge kann eine sogenannte Spondylolisthesis - ein Wirbelgleiten - auftreten. Hierbei handelt es sich um das Gleiten eines Wirbelkörpers nach ventral (Anterolisthese).

Viele Patienten mit Spondylolysen und auch -listhesen haben keine Beschwerden. Beim Sprungschanzenphänomen folgt der Dornfortsatz des betroffenen Wirbels nicht dem nach ventral geglittenen Wirbelkörper. Es ist entsprechend eine Stufe zwischen ihm und dem nächst höheren Dornfortsatz zu tasten. Im fortgeschrittenen Grad der Spondylolisthesis kann es zu einem völligen Abgleiten kommen (Spondyloptose).

Im jugendlichen Alter kann selten durch Druck von dorsal auf die Cauda equina ein Spasmus der ischiocruralen Muskulatur mit der Folge einer Hüftlendenstrecksteife auftreten. Die überwiegende Anzahl von Patienten berichtet über Kreuzschmerzen. Im fortgeschrittenen Stadium des Gleitvorganges kann eine Ischialgie oder Claudicatio spinalis auftreten.

Spondylolysen sprechen in der Regel gut auf konservative Behandlungsmaßnahmen an. Sinnvoll sind eine vorübergehende Einschränkung der körperlichen Aktivität und Kräftigungsübungen der Rücken- und

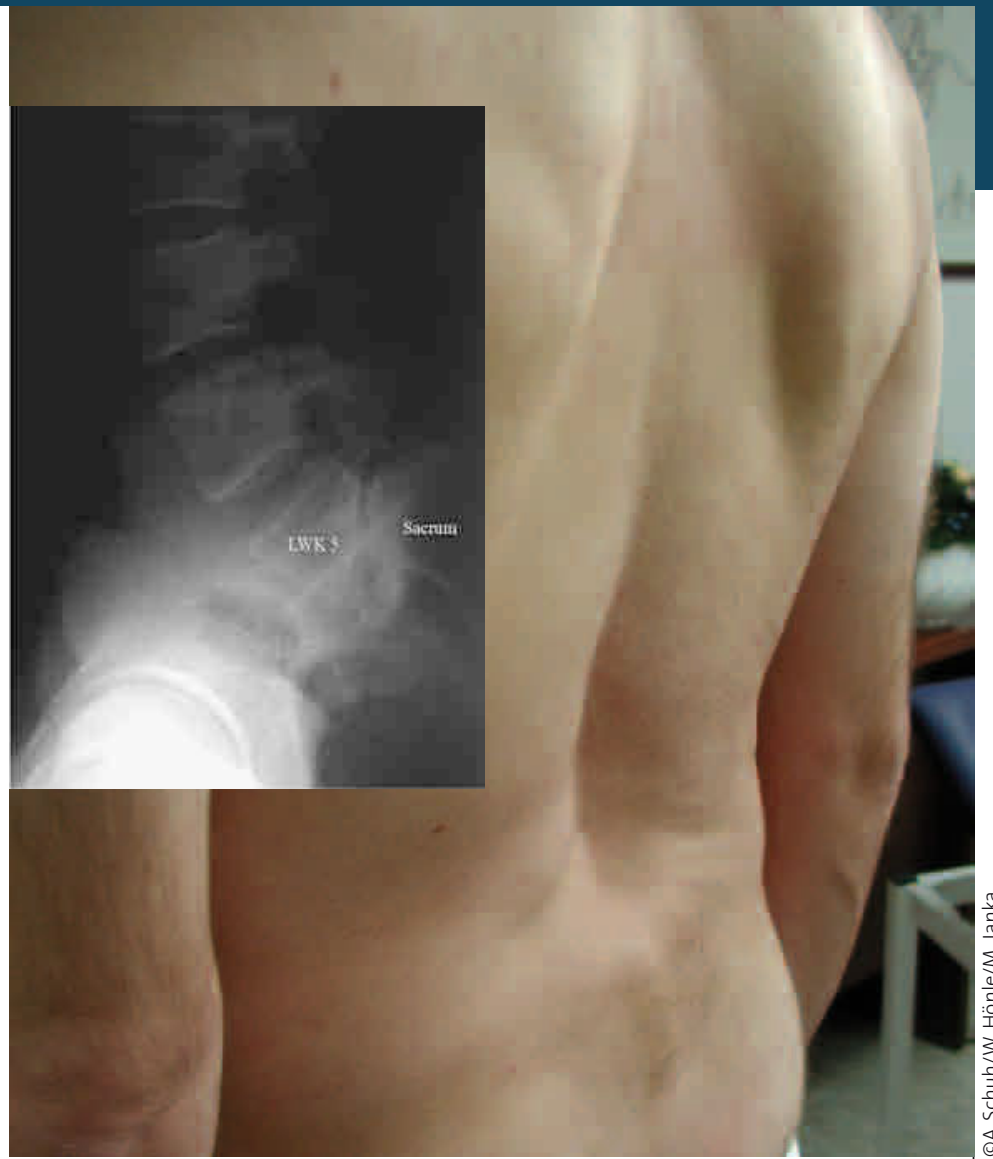

Bauchmuskulatur (entlordosierende Krankengymnastik) und entsprechende analgetisch und antiphlogistische Therapie, ggf. Gabe von Muskelrelaxanzien. Mitunter ist die Versorgung mit Miedern oder Korsetten stadiengerecht indiziert oder eine stationäre Rehabilitationsmaßnahme. Bei der symptomatischen Spondylolisthese, die sich nicht durch konservative Therapiemaßnahmen bessern lässt, insbesondere bei der Claudicatio spinalis, Hüftlendenstrecksteife oder Nervenwurzelreizungen (bis zur Parese) ist die dorsoventrale Spondylodese indiziert.

Keywords. Spondylolisthesis

PD Dr. med. habil. Alexander Schuh (1),

Dr. med. Wolfgang Hönle ${ }^{(2)}$

Dr. med. Michael Janka(3)

(1) Research Unit, Klinikum Neumarkt

(2) Klinik für Orthopädische Chirurgie, Klinikum Neumarkt

(3) Klinik für Wirbelsäulenchirurgie, Klinikum Neumarkt

Akademisches Lehrkrankenhaus der Friedrich-Alexander-Universität Erlangen-

Nürnberg, Nürnberger Straße 12, D-92318 Neumarkt i.d. OPf. 